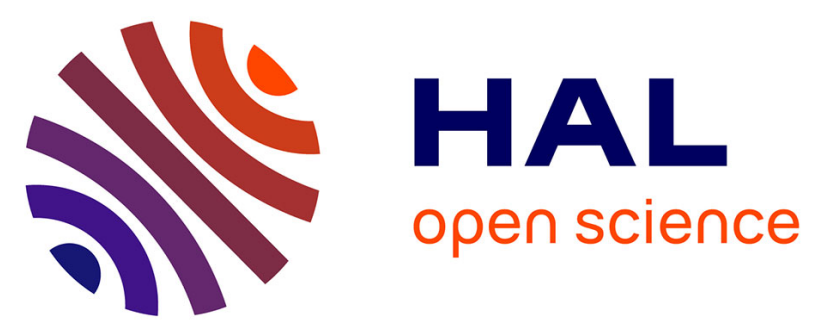

\title{
A model-strengthened imaging biomarker for survival prediction in EGFR-mutated non-small-cell lung carcinoma patients treated with tyrosine kinase inhibitors
}

Annabelle Collin, Vladimir Groza, Louise Missenard, François Chomy, Thierry Colin, Jean Palussière, Olivier Saut

\section{To cite this version:}

Annabelle Collin, Vladimir Groza, Louise Missenard, François Chomy, Thierry Colin, et al.. A modelstrengthened imaging biomarker for survival prediction in EGFR-mutated non-small-cell lung carcinoma patients treated with tyrosine kinase inhibitors. Bulletin of Mathematical Biology, 2021, 83 (6), 10.1007/s11538-021-00902-7 . hal-03428532

HAL Id: hal-03428532

https://hal.inria.fr/hal-03428532

Submitted on 15 Nov 2021

HAL is a multi-disciplinary open access archive for the deposit and dissemination of scientific research documents, whether they are published or not. The documents may come from teaching and research institutions in France or abroad, or from public or private research centers.
L'archive ouverte pluridisciplinaire HAL, est destinée au dépôt et à la diffusion de documents scientifiques de niveau recherche, publiés ou non, émanant des établissements d'enseignement et de recherche français ou étrangers, des laboratoires publics ou privés. 


\title{
A model-strengthened imaging biomarker for survival prediction in EGFR-mutated non-small-cell lung carcinoma patients treated with tyrosine kinase inhibitors
}

\author{
Annabelle Collin · Vladimir Groza · Louise \\ Missenard · François Chomy · Thierry Colin . \\ Jean Palussière . Olivier Saut
}

Received: date / Accepted: date

\author{
Abstract Non-small-cell lung carcinoma is a frequent type of lung cancer with a bad \\ prognosis. Depending on the stage, genomics, several therapeutical approaches are \\ used. Tyrosine Kinase Inhibitors (TKI) may be successful for a time in the treatment \\ of EGFR-mutated non-small cells lung carcinoma. Our objective is here to propose \\ a survival assessment as their efficacy in the long run is challenging to evaluate. The \\ study includes 17 patients diagnosed as of EGFR-mutated non-small cell lung can- \\ cer and exposed to an EGFR-targeting TKI with 3 computed tomography (CT) scans \\ of the primitive tumor (one before the TKI introduction and two after). An imaging \\ biomarker based on the texture heterogeneity evolution between the first and the third \\ exams is derived and computed from a mathematical model and patient data. Defin- \\ A. Collin \\ Univ. Bordeaux, CNRS, Bordeaux INP, IMB, UMR 5251, INRIA Monc, F-33400, Talence, France \\ E-mail: Annabelle.Collin@inria.fr \\ V. Groza \\ Univ. Bordeaux, CNRS, Bordeaux INP, IMB, UMR 5251, INRIA Monc, F-33400, Talence, France \\ E-mail: vladimir.groza@gmail.com \\ L. Missenard \\ Institut Bergonié, F-33076 Bordeaux, France \\ E-mail: louise.missenard@gmail.com \\ F. Chomy \\ Institut Bergonié, F-33076 Bordeaux, France \\ E-mail: F.Chomy@bordeaux.unicancer.fr \\ T. Colin \\ Sophia Genetics, Cité de la Photonique, F-33600, Pessac, France \\ E-mail: TColin@sophiagenetics.com

\section{J. Palussière} \\ Institut Bergonié, F-33076 Bordeaux, France \\ E-mail: J.Palussiere@bordeaux.unicancer.fr \\ O. Saut \\ Univ. Bordeaux, CNRS, Bordeaux INP, IMB, UMR 5251, INRIA Monc, F-33400, Talence, France \\ E-mail: Olivier.Saut@inria.fr
}


ing the overall survival as the time between the introduction of the TKI treatment and the patient death, we obtain a statistically significant correlation between the overall survival and our imaging marker $(p=0.009)$. Using the ROC curve, the patients are separated into two populations and the comparison of the survival curves is statistically significant $(p=0.025)$. The baseline exam seems to have a significant role in the prediction of response to TKI treatment. More precisely, our imaging biomarker defined using only the CT scan before the TKI introduction allows to determine a first classification of the population which is improved over time using the imaging marker as soon as more CT scans are available. This exploratory study leads us to think that it is possible to obtain a survival assessment using only few CT scans of the primary tumor.

Keywords EGFR · TKI · Imaging biomarker · Survival assessment · Mathematical modeling

\section{Introduction}

Tyrosine Kinase Inhibitors (TKI) were shown to be effective in the treatment of EGFR-mutated non-small cell lung carcinoma (NSCLC) [10]. They are currently used as first-line treatment for patients of stage IV. The EGFR gene corresponds to the Epidermal Growth Factor receptor, which belongs to the family of receptors with tyrosine kinase activity. The alteration of the EGFR gene in lung cancer occurs in 5 to $30 \%$ of cases, depending on the patient origin ( $10 \%$ of Caucasian patients, $40 \%$ of non-smoking Caucasian patients and up to $60 \%$ of non-smoking Asian patients), see [3]. TKI treatment may be successful for a time but their efficacy in the long run is challenging to evaluate. For example, in [12], the authors estimate the relapse median time at 10 months.

Latest advances in oncology and the discovery of many different sub-types of cancer, partly because of genomic alterations, open the way to a personalized medicine [22]. There is a need of new tools combining different types of available data to help to choose the best treatment for each patient. Medical imaging has an important role to play in this context as these patients are routinely monitored using CT scans. The most commonly used CT scan evaluation criteria - in particular for lung cancers - is the RECIST (Response Evaluation Criteria In Solid Tumors) which consists in measuring the largest diameters of target lesions $[7,19]$. Some recent studies have shown the advantage of considering tumor volume which is more precise and has a better reproducibility in particular concerning the evaluation of non-small cell lung cancers $[13,14,21]$.

On EGFR-mutated non-small cells lung carcinoma, previous works have studied correlations between the initial shrinking of the primary tumor and the overall survival. One can find contradictory results in the literature: in $[18,20]$, a significant correlation has been established but more recently in [17] this correlation has not be validated using another database. Many recent studies propose to use radiomic approaches which consist in extracting a large number of quantitative features from medical images. In non-small cell lung cancers, various imaging heterogeneity markers may be computed through texture analysis, radiomics, see [25] for a proposal 
for harmonization of methodology. Then, they can be related for example to the distant metastasis probability [27] ; to predict pathological response after neoadjuvant chemoradiation [5] ; to indicate tumor response to radiation therapy [8] ; to advance clinical decision-making by analyzing standard-of-care medical images [11] and to establish independent marker of survival time [9]. In [1], the authors even show that radiomics may help identify a general prognostic phenotype existing in both lung and head-and-neck cancer. These approaches have also been used for EGFR-mutated non-small cell lung cancer. For example, radiomic approaches may predict EGFR mutation status without requiring repeated biopsy acquisitions $[15,26]$ but also identify tumor heterogeneity markers which can be related to early EGFR TKI failure [23].

In this study, instead of testing classical imaging heterogeneity features (e.g. kurtosis, skewness...), we introduce a novel imaging biomarker that quantifies the evolution of the heterogeneity of the primitive tumor of patients with EGFR-mutated non-small cells lung cancers over time. This criterion is derived using our knowledge of the disease and can be biologically interpreted. The objective of this work is to study the value of this novel marker to predict overall survival in order to help clinicians to early detect EGFR TKI treatment failure.

\section{Materials and methods}

\subsection{Patients and clinical context}

A monocentric retrospective cohort study has been conducted on patients with a biopsy-proven non-small-cell lung carcinoma - presenting an identified or suspected EGFR (epidermal growth factor receptor) mutation, (established by a TKI clinical benefit of more than 6 months) - which are non-accessible to local treatment (stage IIIB or IV). Patients were included in the study if 3 CT-scans were available: one at baseline before the first introduction of TKI treatment and two after. The study was approved by Institut Bergonié and IRB approval was obtained for use of the CT images. Informed consents of data collection were waived for research from each patient, in accordance with the related policy of Institut Bergonié.

All patients were exposed to an EGFR-targeting TKI. Two molecules were used: gefitinib (IRESSA, Astra-Zeneca) and erlotinib (TARCEVA, Roche). These two therapies were given until progression, unacceptable toxicity, patient refusal to continue treatment or death.

\subsection{Imaging follow-up and computative biomarkers}

Evaluation scans were done every 2 to 6 months. Each acquisition is performed after an injection of iodized contrast agent at portal phase on the thorax, the abdomen and the pelvis and then at late-arterial phase on the encephalon.

In this work, for each patient, we consider $3 \mathrm{CT}$-scans (acquired at times $t_{0}, t_{1}$ and $t_{2}$ ). The first one $t_{0}$, baseline, is acquired before treatment and the second and the 
third ones are the 2 first ones after the first introduction of TKI treatment. An example for a patient is shown on Fig. 1. Tumors were delineated using a semi-automatic segmentation library that relies on a deformable model [16]. All the segmentations were validated by a junior (LM) and a senior (JP) radiologists.

Fig. 1 Evolution of the tumor of a patient from the study as shown on CT. The lesion is marked by a red arrow on baseline scan, its volume is decreasing under treatment in the following exams. The second and third exams were acquired 117 days and 425 days after the first one respectively.
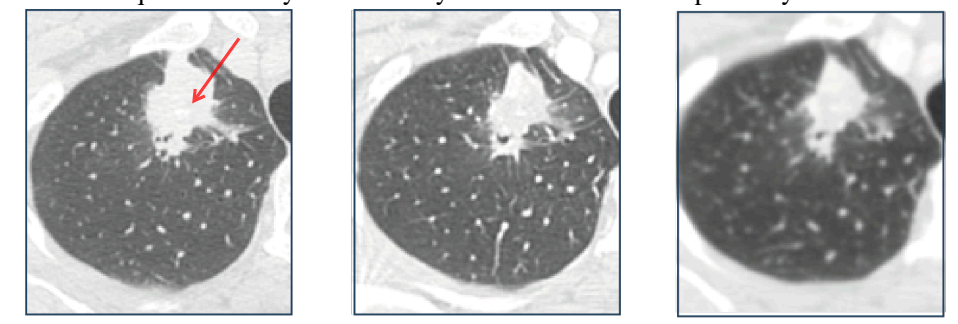

Using these patient images, we derive various biomarkers. Denoting by $V(t)$ the volume of the tumor at time $t$, we define the following vector of volume-based biomarkers:

$$
c_{v}=\left(\frac{V\left(t_{1}\right)-V\left(t_{0}\right)}{t_{1}-t_{0}}, \frac{V\left(t_{2}\right)-V\left(t_{0}\right)}{t_{2}-t_{0}}, \frac{V\left(t_{1}\right)}{V\left(t_{0}\right)}, \frac{V\left(t_{2}\right)}{V\left(t_{0}\right)}\right)^{T},
$$

corresponding respectively to the initial slope of volume between $t_{0}$ and $t_{1}$, to the slope of volume between $t_{0}$ and $t_{2}$, to the initial ratio of volume between $t_{0}$ and $t_{1}$ and to the total ratio of volume between $t_{0}$ and $t_{2}$.

On CT, high intensities (white color on the image) correspond to high tissue densities and therefore to high cellularity while smaller intensities around 0 and below on Hounsfield scale correspond to e.g. lung parenchyma, air, water or necrosis. For this reason, we choose to split the set of voxels within the tumor into two classes. The first class contains the voxels whose values in Hounsfield units are non-negative while the second class is formed by non-positive voxels. We will refer to the first class (with non-negative intensities) as being the proliferative one while the second one will be referred as the non-proliferative one. Using this decomposition, we aim at taking tumor heterogeneity (very roughly) into account without requiring complex models that would be difficult to calibrate. We denote by $P(t)$ the volume of the set of voxels whose intensities are positive within the tumor on the exam at time $t$. We then compute the ratio of this proliferative-like compartment with respect to the total volume $\% P(t)=\frac{P(t)}{V(t)}$. We derive the following vector of heterogeneity biomarkers:

$$
c_{h}=\left(\int_{t_{0}}^{t_{2}} \% P(t) d t, \int_{t_{1}}^{t_{2}} \% P(t) d t, \% P\left(t_{0}\right)\right)^{T},
$$

corresponding respectively to the area under the curve (AUC) of the quantity $\% P(t)$ between $t_{0}$ and $t_{2}$, to the AUC of the quantity $\% P(t)$ between $t_{1}$ and $t_{2}$, and to the initial value of the quantity $\% P(t)$. 
Using the first 3 CT-scans, we extract $V\left(t_{0}\right), V\left(t_{1}\right), V\left(t_{2}\right), P\left(t_{0}\right), P\left(t_{1}\right)$ and $P\left(t_{2}\right)$. One strategy to approximate the imaging marker $\int_{t_{0}}^{t_{2}} \% P(t) d t$ consists in applying the trapezoidal rule using extracted data to approximate the integral. However, this strategy is not robust enough and too sensitive to the time intervals variability. Indeed, the acquisition times $t_{0}, t_{1}$ and $t_{2}$ and the CT image noise may introduce some instability in the computation (for example if $t_{1}$ is too close to $t_{0}$ or $t_{2}$ ). In addition, the linear interpolation between sampling points cannot represent accurately the actual behavior of the ratio of the proliferative-like compartment (see Section 3 for an illustrative example). As a consequence, the trapezoidal rule does not seem to be an acceptable method and one has to find another approach to estimate these quantities reliably.

2.3 Improving the robustness of the biomarker computation using a mechanistic model

To improve the robustness and the accuracy of our computative biomarker $\int_{t_{0}}^{t_{2}} \% P(t) d t$, we choose another strategy that consists in using a mechanistic model to estimate the evolution of $P$ continuously on the time interval $\left[t_{0}, t_{2}\right]$. More precisely, this model describes the evolutions of two populations of cells (proliferative cells and nonproliferative compartments) and its personalized parameters are recovered using the quantities $V\left(t_{0}\right), V\left(t_{1}\right), V\left(t_{2}\right), P\left(t_{0}\right), P\left(t_{1}\right)$ and $P\left(t_{2}\right)$ defined in the previous section. Hence an evaluation of $P(t)$ and $V(t)$ at any time between $t_{0}$ and $t_{2}$ is computed and used to estimate our biomarker.

Fig. 2 Modeling of temporal evolution of proliferative and non-proliferative cells.

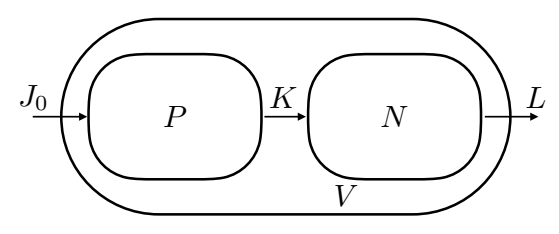

\begin{tabular}{l}
\hline$P$, density of proliferative cells \\
$N$, density of non-proliferative cells \\
\hline$J_{0}$, growth rate \\
$K$, decreasing rate due to TKI treatment \\
$L$, evacuation rate due to TKI treatment \\
\hline
\end{tabular}

Let us now describe the derivation of the model. We denote by $P$ (resp. by $N$ ) the volume of proliferative (resp. quiescent or non-proliferative) cells. We have $P+N=$ $V$ where $V$ corresponds to the volume of the lesion. The evolution of the volume of proliferative cells is supposed to satisfy the following equation:

$$
P^{\prime}=\left(J_{0}-K\right) P
$$

where $P^{\prime}$ denotes the time-derivative of $P$, the quantity $J_{0}$ is the growth rate and we assume it constant in order to keep a model with identifiable parameters. The quantity $K$ corresponds to the decreasing rate due to the TKI treatment. We assume that it follows a Gompertz-like law:

$$
K=K_{0} e^{-\delta t}
$$


where $\delta$ is an unknown parameter and $K_{0}$ the initial decreasing rate of $P$. We assume that when under treatment, proliferative cells die and enter the non-proliferative compartment. The evolution of the density $N$ of this non-proliferative compartment is supposed to satisfy the following equation:

$$
N^{\prime}=K P-L N .
$$

The quantity $L$ is the evacuation (or metabolization) rate of the non-proliferative compartment. We assume that it follows a Gompertz-like law:

$$
L=L_{0} e^{-\eta t}
$$

where $\eta$ is an unknown parameter and $L_{0}$ the initial evacuation rate of $N$.

This leads to the following ordinary differential system

$$
\left\{\begin{array}{l}
P^{\prime}=\left(J_{0}-K\right) P \\
N^{\prime}=K P-L N \\
K=K_{0} e^{-\delta t} \\
L=L_{0} e^{-\eta t}
\end{array}\right.
$$

in which the quantity $P$ can be explicitly determined:

$$
P=P_{0} e^{\frac{K_{0}}{\delta}\left(e^{-\delta t}-1\right)-N_{0} t}
$$

and the quantity $N$ can be numerically approximated. Figure 2 illustrates this model in the formalism of compartment models.

To be used on patient data, this model has to be calibrated i.e. the values of its parameters have to be recovered from the observations. The model has 5 parameters: $K_{0}, \delta, J_{0}, L_{0}$ and $\eta$ that we want to estimate using (the subscript $d$ is used to denote observations):

- the volumes $V_{d}\left(t_{1}\right)$ and $V_{d}\left(t_{2}\right)\left(V_{d}\left(t_{0}\right)\right.$ is used for the initial condition),

- the proliferative parts $P_{d}\left(t_{1}\right)$ and $P_{d}\left(t_{2}\right)\left(P_{d}\left(t_{0}\right)\right.$ is used for the initial condition).

The parametrization is obtained in two steps. We start by estimating $K_{0}, J_{0}$ and $\delta$ by minimizing

$$
\mathscr{E}_{P}=\sqrt{\sum_{i=1,2}\left|P_{d}\left(t_{i}\right)-P\left(t_{i}\right)\right|^{2}} .
$$

Two cases are possible and give priors on some of the parameters:

- Case $P_{d}\left(t_{0}\right)>P_{d}\left(t_{1}\right)>P_{d}\left(t_{2}\right)$ : we assume that the density of proliferative cells is decreasing and we search the parameters as

$$
\left.\left.\left.K_{0} \in\right] 0,0.1\right], N_{0} \in\right] 0, K_{0}[\text { and } \delta \in]-t_{2} \ln \left(N_{0} / K_{0}\right), t_{2} \ln \left(N_{0} / K_{0}\right)[\text {. }
$$

- Case $P_{d}\left(t_{0}\right)>P_{d}\left(t_{1}\right)$ and $P_{d}\left(t_{1}\right)<P_{d}\left(t_{2}\right)$ : we search the parameters as:

$$
\left.\left.\left.K_{0} \in\right] 0,0.1\right], N_{0} \in\right] 0, K_{0}[\text { and } \delta \in]-0.1,0.1[\text {. }
$$


The set of parameters is not unique. Yet, if two different sets of parameters give small errors $\left(\right.$ e.g. $\left.\sim 10^{-3}\right)$, the variations of $P$ for each of these two sets should be quite similar and the values of our biomarker would not differ by much. The second step consists in estimating $L_{0}$ and $\eta$ by minimizing

$$
\mathscr{E}_{V}=\sqrt{\sum_{i=1,2}\left|V_{d}\left(t_{i}\right)-V\left(t_{i}\right)\right|^{2}} .
$$

As we consider that the TKI treatment is still acting (for all the patients, we have $\left.V_{d}\left(t_{0}\right)>V_{d}\left(t_{1}\right)>V_{d}\left(t_{2}\right)\right)$, the sets of parameters for which $V$ is not strictly decreasing are rejected.

\subsection{Derivation from a spatial model}

This is of no direct use for the evaluation of relapse but this ODE system can be derived by integration of a spatial model over the tumor domain. Let us denote by $\hat{P}$ (resp. $\hat{N})$ the spatial density of proliferative (resp. non-proliferative or quiescent) cells and by $\vec{v}$, the velocity field that describes the evolution of the tumor over time. Following $[2,4,24]$, the tumor can be described by the evolution in space and time of population of $\hat{P}$ and $\hat{N}$,

$$
\left\{\begin{array}{l}
\partial_{t} \hat{P}+\nabla \cdot(\vec{v} \hat{P})=J_{0} \hat{P}-K \hat{P} \\
\partial_{t} \hat{N}+\nabla \cdot(\vec{v} \hat{N})=K \hat{P}-L \hat{N} \\
M=K_{0} e^{-\delta t} \\
L=L_{0} e^{-\eta t} \\
\nabla \cdot \vec{v}=J_{0} \hat{P}-L \hat{N} \\
\vec{v}=-\nabla \pi
\end{array}\right.
$$

where the last equation closes the system using a Darcy law, where $\pi$ corresponds to a pressure or potential. Using Reynolds theorem, this system of partial differential equations is related to System (1) by

$$
P(t)=\int_{\Omega(t)} \hat{P}(t, x) d x \text { and } Q(t)=\int_{\Omega(t)} \hat{Q}(t, x) d x,
$$

where $\Omega$ is the tumor domain. The PDE model and the ODE ones share the same parameters (besides initial conditions of the scalar fields).

\subsection{Statistics}

Whenever appropriate, standard statistics are presented as mean \pm standard-deviation and number (percentage). We define the overall survival (OS) as the time between the introduction of the TKI treatment and the patient death. Survival curves were computed using the Kaplan-Meier estimator and compared using Log-Rank tests. The association of survival failure with each investigated biomarker was tested using Cox regression. Prediction performances of the biomarkers were assessed using ROC 
curves. The appropriate statistical tests were performed when required with a significance threshold set to $p=0.05$. The mechanistic model was fitted using a classical MCMC method (with the priors from the previous section). All computations were performed using Matlab-R2015a.

\section{Results}

A population of 25 clinical cases has been collected at Institut Bergonié (Bordeaux, France) between years 2006 and 2013. Among them, only 17 patients were kept: we have excluded 2 cases for which the CT-scan before the TKI introduction was not available and 6 cases for which it was not possible to delineate the tumor (miliary disease, patients without any discernible lesion e.g. with pleural effusion or atelectasis). Table 1 presents the patient cohort: age, sex, smoking information, mutation, stage and if the patient had a treatment before the TKI introduction.

Table 1 Presentation of the patient cohort: sex, age, smoking, mutation, stage, previous treatment before the TKI introduction.

\begin{tabular}{|c|c|c|}
\hline \multicolumn{3}{|c|}{ Characteristics } \\
\hline \multirow{2}{*}{ Sex } & Women & $14(82 \%)$ \\
\cline { 2 - 3 } & Men & $3(18 \%)$ \\
\hline Age & & $65 \pm 11$ \\
\hline \multirow{3}{*}{ Smoking } & Yes & $1(6 \%)$ \\
\cline { 2 - 3 } & No & $10(59 \%)$ \\
\cline { 2 - 3 } & Unknown & $6(35 \%)$ \\
\hline \multirow{3}{*}{ Mutation } & Exon 19 & $7(41 \%)$ \\
\cline { 2 - 3 } & Exon 21 & $7(41 \%)$ \\
\cline { 2 - 3 } & Exon 18 & $1(6 \%)$ \\
\cline { 2 - 3 } & Unknown & $2(12 \%)$ \\
\hline \multirow{2}{*}{ Stage } & IV & $16(94 \%)$ \\
\cline { 2 - 3 } & IIIB & $1(6 \%)$ \\
\hline \multirow{2}{*}{ Previous treat. } & No & $11(65 \%)$ \\
\cline { 2 - 3 } & Yes & $6(35 \%)$ \\
\hline
\end{tabular}

The evolutions of the densities of proliferative (red) and non-proliferative (green) cells for the 17 patients with the evolution of the volume (in blue) are presented in Fig. 3. The $\mathrm{x}$-axis represents the time in days, while the volume is reported on the $y$-axis. The blue points show the values of the volume at time and while the red and green point are respectively the volume of proliferative and non-proliferative compartments at the same time point. The blue (resp. red, green) curve describes the evolution of the volume (resp. density of proliferative cells, density of non-proliferative cells) given by the mechanistic model and the best set of parameters fitting observations

The evolution of the ratio $\% P(t)=\frac{P(t)}{V(t)}$ of the proliferative-like compartment with respect to the total volume for one patient is presented in Figure 4. This gives an illustrative example of the limitation of using a linear interpolation of sampling data to compute $\int_{t_{0}}^{t_{2}} \% P(t)$ regarding the accuracy of the evolution of $\% P$. 
Fig. 3 : Trajectories of the personalized model: plain lines show evolutions of the densities of proliferative (red) and non-proliferative (green) cells for 17 patients with the evolution of the volume (in blue). Blue dots represent measurements of tumor volumes from patients exams performed by a senior radiologists. Red and green dots are proliferative (resp. non-proliferative) densities computed directly from the observations. Overall survival (OS) is shown for each patient above the corresponding figure.
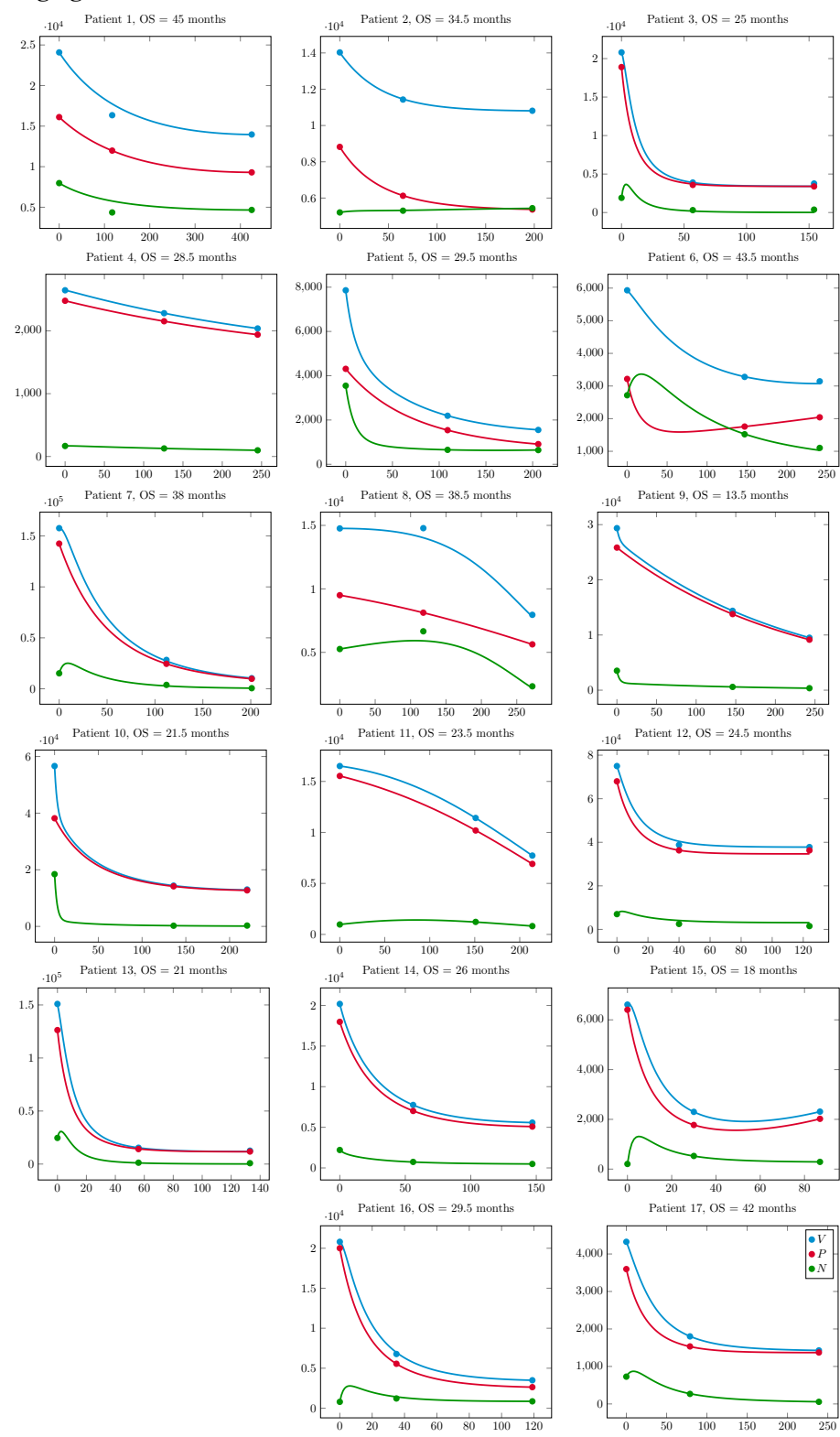

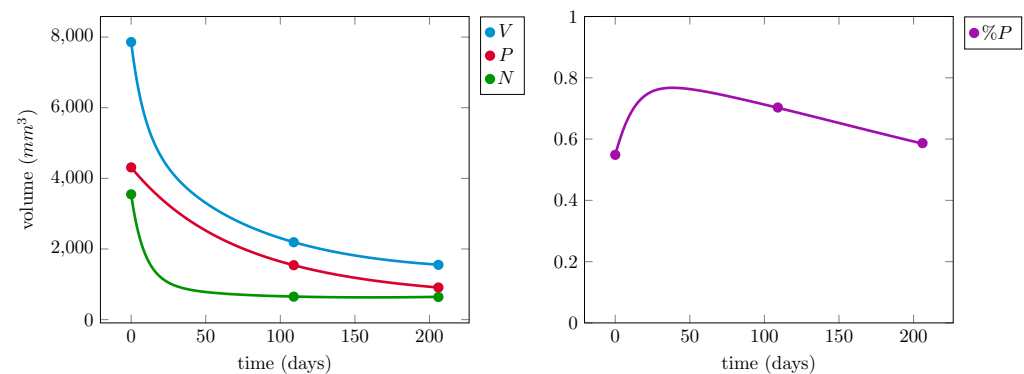

Fig. 4 Trajectories of the personalized model for Patient 5. Left: evolutions of the densities of proliferative (red) and non-proliferative (green) cells with the evolution of the volume (in blue). Right: evolution of the ratio $\% P(t)=\frac{P(t)}{V(t)}$ of the proliferative-like compartment with respect to the total volume.

First of all, there seems to be no significant correlation between the individual volume-based biomarkers gathered in the vector

$$
c_{v}=\left(\frac{V\left(t_{1}\right)-V\left(t_{0}\right)}{t_{1}-t_{0}}, \frac{V\left(t_{2}\right)-V\left(t_{0}\right)}{t_{2}-t_{0}}, \frac{V\left(t_{1}\right)}{V\left(t_{0}\right)}, \frac{V\left(t_{2}\right)}{V\left(t_{0}\right)}\right)^{T},
$$

and the overall survival ( $p=0.48, p=0.36, p=0.23$ and $p=0.17$ respectively).

Let us now focus on the heterogeneity-based biomarkers gathered in the vector

$$
c_{h}=\left(\int_{t_{0}}^{t_{2}} \% P(t) d t, \int_{t_{1}}^{t_{2}} \% P(t) d t, \% P\left(t_{0}\right)\right)^{T} .
$$

The values of the first heterogeneity-based biomarker

$$
\int_{t_{0}}^{t_{2}} \% P(t) d t=\int_{t_{0}}^{t_{2}} \frac{P(t)}{V(t)} d t
$$

for all the patients are plotted in Fig. 5.

Fig. 5 Imaging marker $\int_{t_{0}}^{t_{2}} \% P(t) d t$ after normalization (y-axis) versus overall survival in months $(x$-axis) for each patient (black dots). Linear regression is shown with the blue line.

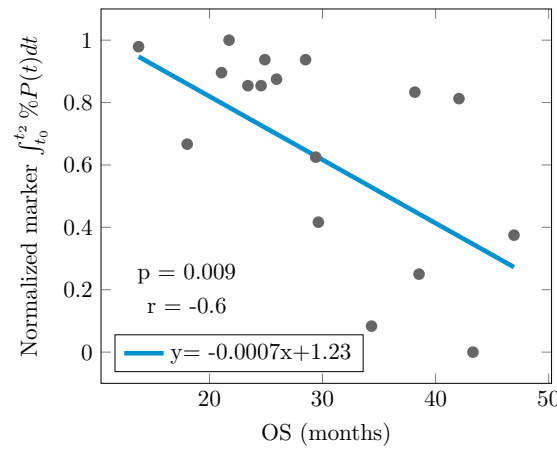


The values of the criteria based on the evolution of the tumor heterogeneity have been normalized using its maximum value and is reported on the $y$-axis. The $\mathrm{x}$-axis corresponds to the value of the overall survival. The blue curve corresponds to the linear regression. The correlation between the overall survival and the imaging marker is statistically significant ( $p=0.009, r=-0.6$ ). The population may clearly be divided into two populations. In particular, patients with a short survival time have a large value of the biomarker. In order to find the best threshold to classify the patients, we need to set a survival threshold. Based on the survival histogram of the 17 patients given in Fig. 6 and for clinical relevance, we set the survival threshold at 30 months.

Fig. 6 Survival histogram (17 patients). $x$-axis represents survival time (in months), $y$-axis frequency (in number of patients).

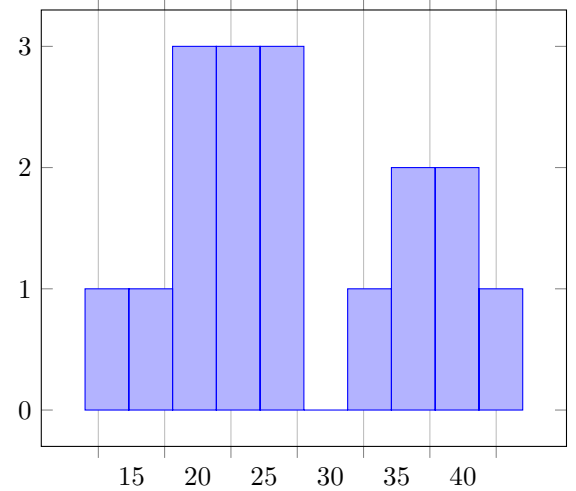

The ROC curve (AUC $=0.81$ ) is given in Fig. 7 (see blue curve). Using Fig. 7 , we see that a good trade-off between sensibility and specificity consists in taking a normalized threshold for the biomarker of 0.4 that is optimal with a sensibility of 0.9 and a specificity of 0.7 .

Fig. 7 ROC curve of the imaging marker $\int_{t_{0}}^{t_{2}} \% P(t) d t$ (after normalization).

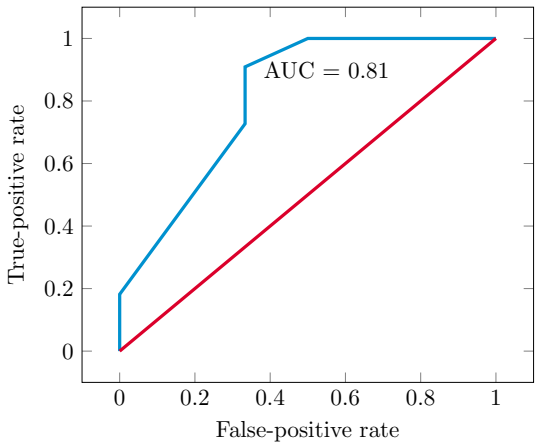


Finally, survival curves are given in Fig. 8: in red the survival curve of the full population and in blue (resp. in green) the survival curve of the population with a short (resp. with a large) imaging marker. The normalized threshold value 0.4 obtained with the ROC curve is used to separate the patients. The comparison of the survival curves of these two populations is statistically significant $(p=0.025$, hazard ratio $=0.25$ with a $95 \%$ confidence interval equals to $0.09-0.7)$.

Fig. 8 : Survival curves (full population in red ; patients with a small (resp. large) value of the imaging marker $\int_{t_{0}}^{t_{2}} \% P(t) d t$ in blue (resp. in green).

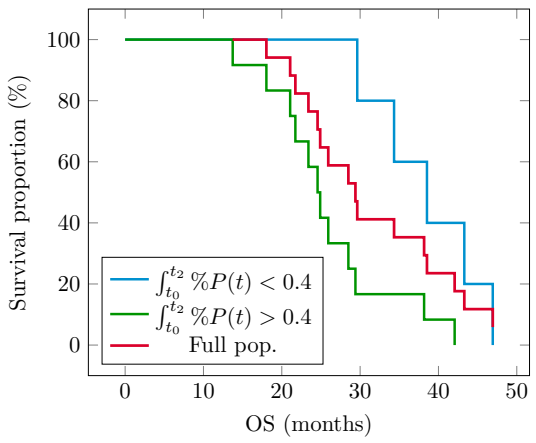

However, the strategy is not efficient if we only use the time average between $t_{1}$ and $t_{2}$ to define the imaging biomarker. Indeed there is no correlation between the second heterogeneity-based biomarker $\int_{t_{1}}^{t_{2}} \% P(t) d t$ and the overall survival $(\mathrm{p}=$ $0.34)$. This means that the initial status of the tumor has a major role for the prognosis of the response to the TKI treatment. More precisely, there is a significant correlation between the third heterogeneity-based biomarker $\% P\left(t_{0}\right)$ and the overall survival $(p=0.034)$ even if the biomarker based on the 3 CT-exams is six times more significant ( $p=0.009$ ), see Fig. 9. Concerning the survival curves, we obtain equivalent results $(p=0.036$ instead of $p=0.025)$.

Fig. 9 : Left - Imaging biomarker $\% P\left(t_{0}\right)$ versus overall survival. Middle - ROC curve. Right Survival curves (the threshold value has been fixed using the ROC curve).
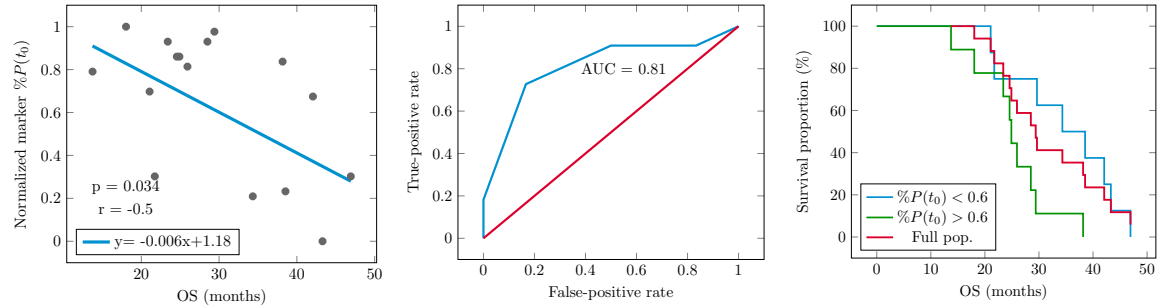


\section{Discussion}

In this work we have studied the ability of volume-based and heterogeneity-based imaging biomarkers to estimate the survival in EGFR-mutated NSCLC patients treated with TKI from early imaging follow-up.

Our first result is that there seems to be no correlation between volume-based imaging biomarkers and survival. This finding is consistent with the work [17] who noticed a lack of association between tumor shrinkage and long-term survival. This illustrates why the response to TKI treatment is difficult to estimate using only the evolution of tumor volume (or RECIST).

Our second result is that heterogeneity-based imaging biomarkers may help predict short-term survival. More precisely, we propose an imaging biomarker $\int_{t_{0}}^{t_{2}} \% P(t) d t$ that is able to discriminate patients with short-time survival using only 3 successive CT-scans (an imaging monitoring performed as standard of care for such patients). This biomarker is computed through a personalized mechanistic model to interpolate between time points. To the best of our knowledge, this is the first study in which a mechanistic model based on disease knowledge has been used to predict the outcome in EGFR-mutated NSCLC patients treated with TKI. This shows that characterizing the content of the tumor and its dynamics using mathematical models might provide valuable information to guide clinical decisions. As a clinical consequence - assuming the biomarker is validated at a reasonable scale - patients predicted to have a low survival (e.g. patients with a biomarker value under 0.4 as in Fig. 8), could have their treatment changed before the end of the complete TKI administration.

The main strength of this work lies in the fact that the imaging marker is based on a mathematical description of the alleged underlying pathophysiological processes rather than purely empirical observations. As a consequence, the value of the biomarker may be given a phenomenological meaning, an interpretation that would be lacking otherwise. A large value of the biomarker means that the proportion of proliferative cells does not decrease over time (even if the volume of the lesion is decreasing). In the opposite case, a small value of the marker implies a decrease of the proportion of the proliferative compartment, even if the response is modest in terms of whole tumor size.

We show that the use of the first image (baseline CT-scan acquired before TKI treatment) is of paramount importance for a biomarker to predict the survival. More precisely, the imaging biomarker computed using only this first image provides a first classification of the patient that can be incrementally improved using the imaging marker as more follow-up CT-scans become available.

In addition to biomarkers, the mathematical model offers more information on the mechanism of the response to treatment. The evolutions of the proliferative and nonproliferative compartments given for each patient might also be useful to personalize therapy, see Figure 3. In particular, it would be interesting to study the ability of this mathematical model to detect acquired resistances to TKI earlier, especially T790M mutation, a feature associated with bad prognostic [6].

A major limitation of this work is the small sample size (which limits the power of the statistical analysis and does not allow validating on cases not used to set the biomarker threshold) and its retrospective nature. The small size of the dataset also 
prevents us to perform any serious uncertainty quantification or to increase the dimensionality of biomarkers (one could also consider jointly biological or clinical features). A second limitation concerns the assumptions of the mechanistic model: the tumor imaging heterogeneity as measured using CT-scan is assumed to be related to the proportion of proliferative and non-proliferative cells. Confirming this hypothesis would require a histological assessment of the whole tumor, which is possible only in patients who undergo surgery. Yet we believe that the results are still interesting and are worthy of being validated with a larger cohort (work is ongoing in this direction). The mechanistic is also simplistic in order to be calibrated on sparse data. The mechanisms at stake that are responsible for tumoral response to treatment are barely reproduced. If more information was available, one should definitely improve this modeling to have a more accurate model and eventually a better survival evaluation. This was not the case in our study but work is also ongoing on adding clinical and omics data to imaging.

\section{Conclusion}

We have shown that the initial volume (or RECIST) evolution under TKI is not sufficient to predict the survival while the tumor heterogeneity before the TKI introduction is a major prognosis factor and provides a first classification of patients. Furthermore, this first classification can be incrementally improved using the imaging marker that summarizes the early evolution of the tumor heterogeneity as more CT scans become available. Short term perspectives of this work are about increasing the size of the cohort and improving the segmentation process in order to be able to include patients with non-delineated tumors (at this is a tedious task to perform for radiologist that could not be done routinely).

Acknowledgements This study was supported by the French Laboratory of Excellence TRAIL ANR-10LABX-57.

\section{References}

1. Aerts, H.J.W.L., Velazquez, E.R., Leijenaar, R.T.H., Parmar, C., Grossmann, P., Carvalho, S., Cavalho, S., Bussink, J., Monshouwer, R., Haibe-Kains, B., Rietveld, D., Hoebers, F., Rietbergen, M.M., Leemans, C.R., Dekker, A., Quackenbush, J., Gillies, R.J., Lambin, P.: Decoding tumour phenotype by noninvasive imaging using a quantitative radiomics approach. Nature Communications 5, 4006 (2014). DOI 10.1038/ncomms5006

2. Billy, F., Ribba, B., Saut, O., Morre-Trouilhet, H., Colin, T., Bresch, D., Boissel, J.P., Grenier, E., Flandrois, J.P.: A pharmacologically based multiscale mathematical model of angiogenesis and its use in investigating the efficacy of a new cancer treatment strategy. Journal of theoretical biology 260(4), 545-562 (2009)

3. Cadranel, J., Ruppert, A.M., Beau-Faller, M., Wislez, M.: Therapeutic strategy for advanced EGFR mutant non-small-cell lung carcinoma. Critical reviews in oncology/hematology 88(3), 477-493 (2013)

4. Colin, T., Ribba, B., Schnell, S.: A multiscale mathematical model of cancer growth and radiotherapy efficacy: The role of cell cycle regulation in response to irradiation. Theoretical Biology and Medical Modelling p. 3 (2006) 
5. Coroller, T.P., Agrawal, V., Narayan, V., Hou, Y., Grossmann, P., Lee, S.W., Mak, R.H., Aerts, H.J.W.L.: Radiomic phenotype features predict pathological response in non-small cell lung cancer. Radiotherapy and Oncology 119(3), 480-486 (2016). DOI 10.1016/j.radonc.2016.04.004. URL http://www.thegreenjournal.com/article/S0167-8140(16)31038-6/abstract

6. Dong, Y., Zhou, Z., Wang, J., Ma, L., Liu, Z., Wang, Y., Song, J., Zhang, S., Che, N.: Origin of the T790m mutation and its impact on the clinical outcomes of patients with lung adenocarcinoma receiving EGFR-TKIs. Pathology-Research and Practice (2019)

7. Eisenhauer, E.A., Therasse, P., Bogaerts, J., Schwartz, L.H., Sargent, D., Ford, R., Dancey, J., Arbuck, S., Gwyther, S., Mooney, M.: New response evaluation criteria in solid tumours: revised RECIST guideline (version 1.1). European journal of cancer 45(2), 228-247 (2009)

8. Fave, X., Zhang, L., Yang, J., Mackin, D., Balter, P., Gomez, D., Followill, D., Jones, A.K., Stingo, F., Liao, Z.: Delta-radiomics features for the prediction of patient outcomes in non-small cell lung cancer. Scientific reports 7(1), 588 (2017)

9. Ganeshan, B., Panayiotou, E., Burnand, K., Dizdarevic, S., Miles, K.: Tumour heterogeneity in nonsmall cell lung carcinoma assessed by CT texture analysis: a potential marker of survival. European radiology $\mathbf{2 2 ( 4 ) , 7 9 6 - 8 0 2 ~ ( 2 0 1 2 ) ~}$

10. Greenhalgh, J., Dwan, K., Boland, A., Bates, V., Vecchio, F., Dundar, Y., Jain, P., Green, J.A.: Firstline treatment of advanced epidermal growth factor receptor (EGFR) mutation positive non-squamous non-small cell lung cancer. The Cochrane Library (2016)

11. Grossmann, P., Stringfield, O., El-Hachem, N., Bui, M.M., Velazquez, E.R., Parmar, C., Leijenaar, R.T., Haibe-Kains, B., Lambin, P., Gillies, R.J.: Defining the biological basis of radiomic phenotypes in lung cancer. Elife 6 (2017)

12. Helena, A.Y., Sima, C.S., Huang, J., Solomon, S.B., Rimner, A., Paik, P., Pietanza, M.C., Azzoli, C.G., Rizvi, N.A., Krug, L.M.: Local therapy with continued EGFR tyrosine kinase inhibitor therapy as a treatment strategy in EGFR-mutant advanced lung cancers that have developed acquired resistance to EGFR tyrosine kinase inhibitors. Journal of Thoracic Oncology 8(3), 346-351 (2013)

13. Hwang, K.E., Kim, H.R.: Response Evaluation of Chemotherapy for Lung Cancer. Tuberculosis and respiratory diseases $\mathbf{8 0}(2), 136-142$ (2017)

14. Jiang, B., Zhou, D., Sun, Y., Wang, J.: Systematic analysis of measurement variability in lung cancer with multidetector computed tomography. Annals of thoracic medicine 12(2), 95 (2017)

15. Kim, H., Chae, K.J., Yoon, S.H., Kim, M., Keam, B., Kim, T.M., Kim, D.W., Goo, J.M., Park, C.M.: Repeat biopsy of patients with acquired resistance to EGFR TKIs: implications of biopsyrelated factors on T790m mutation detection. European Radiology 28(2), 861-868 (2018). DOI 10.1007/s00330-017-5006-6

16. Lachaud, J.O., Taton, B.: Deformable model with a complexity independent from image resolution. Computer Vision and Image Understanding 99(3), 453-475 (2005)

17. Lee, C.K., Lord, S., Marschner, I., Wu, Y.L., Sequist, L., Rosell, R., Fukuoka, M., Mitsudomi, T., Asher, R., Davies, L., others: The value of early depth of response in predicting long-term outcome in EGFR-mutant lung cancer. Journal of Thoracic Oncology 13(6), 792-800 (2018)

18. Lee, J.H., Lee, H.Y., Ahn, M.J., Park, K., Ahn, J.S., Sun, J.M., Lee, K.S.: Volume-based growth tumor kinetics as a prognostic biomarker for patients with EGFR mutant lung adenocarcinoma undergoing EGFR tyrosine kinase inhibitor therapy: a case control study. Cancer Imaging 16(1), 5 (2016)

19. van Meerten, E.L.v.P., Gelderblom, H., Bloem, J.L.: RECIST revised: implications for the radiologist A review article on the modified RECIST guideline. European radiology 20(6), 1456-1467 (2010)

20. Nishino, M., Dahlberg, S.E., Fulton, L.E., Digumarthy, S.R., Hatabu, H., Johnson, B.E., Sequist, L.V.: Volumetric tumor response and progression in EGFR-mutant NSCLC patients treated with erlotinib or gefitinib. Academic radiology 23(3), 329-336 (2016)

21. Nishino, M., Guo, M., Jackman, D.M., DiPiro, P.J., Yap, J.T., Ho, T.K., Hatabu, H., Jänne, P.A., Van den Abbeele, A.D., Johnson, B.E.: CT tumor volume measurement in advanced non-small-cell lung cancer: performance characteristics of an emerging clinical tool. Academic radiology 18(1), 54-62 (2011)

22. Nishino, M., Jagannathan, J.P., Krajewski, K.M., O'Regan, K., Hatabu, H., Shapiro, G., Ramaiya, N.H.: Personalized tumor response assessment in the era of molecular medicine: cancer-specific and therapy-specific response criteria to complement pitfalls of RECIST. American Journal of Roentgenology 198(4), 737-745 (2012)

23. Park, S., Ha, S., Lee, S.H., Paeng, J.C., Keam, B., Kim, T.M., Kim, D.W., Heo, D.S.: Intratumoral heterogeneity characterized by pretreatment PET in non-small cell lung cancer patients predicts progression-free survival on EGFR tyrosine kinase inhibitor. PloS One 13(1), e0189766 (2018). DOI 10.1371/journal.pone.0189766 
24. Ribba, B., Saut, O., Colin, T., Bresch, D., Grenier, E., Boissel, J.P.: A multiscale mathematical model of avascular tumor growth to investigate the therapeutic benefit of anti-invasive agents. Journal of theoretical biology 243(4), 532-541 (2006)

25. Sollini, M., Cozzi, L., Antunovic, L., Chiti, A., Kirienko, M.: PET Radiomics in NSCLC: state of the art and a proposal for harmonization of methodology. Scientific Reports 7(1), 358 (2017). DOI 10.1038/s41598-017-00426-y

26. Zhang, L., Chen, B., Liu, X., Song, J., Fang, M., Hu, C., Dong, D., Li, W., Tian, J.: Quantitative Biomarkers for Prediction of Epidermal Growth Factor Receptor Mutation in Non-Small Cell Lung Cancer. Translational Oncology 11(1), 94-101 (2018). DOI 10.1016/j.tranon.2017.10.012. URL http://www.transonc.com/article/S1936-5233(17)30261-9/abstract

27. Zhou, H., Dong, D., Chen, B., Fang, M., Cheng, Y., Gan, Y., Zhang, R., Zhang, L., Zang, Y., Liu, Z., Zheng, H., Li, W., Tian, J.: Diagnosis of Distant Metastasis of Lung Cancer: Based on Clinical and Radiomic Features. Translational Oncology 11(1), 31-36 (2018). DOI 10.1016/j.tranon.2017.10.010 URL http://www.transonc.com/article/S1936-5233(17)30320-0/abstract 